\title{
Wie zuverlässig ist die E-Mail-Anwendung?
}

Der E-Mail-Dienst ist die meist genutzte Anwendung im Internet. Ob beruflich oder privat, fast jeder hat eine oder mehrere E-Mail-Adressen und nutzt die schnelle und einfache Kommunikationsmöglichkeit im Internet.

Wir schreiben E-Mails während der Arbeit, von zu Hause, vom Handy aus dem Zug, am Flughafen von unserem Notebook, aus dem Hotel, aus den Internet-Cafés der ganzen Welt, usw. Der Umgang mit den Zugangsmöglichkeiten zum E-MailDienst ist einfach, und der schnelle Austausch von Informationen ist genial und preiswert.

Die Oma schreibt E-Mails an ihre Enkel, unsere Kanzlerin Frau Merkel schickt eine E-Mail an den US-Präsidenten Obama, Schüler verabreden sich zum Kino, Verliebte zum Date, Ackermann schickt eine E-Mail an Schäuble, Mitarbeiter von internationalen Konzernen schicken ihre E-Mails in die ganze Welt, usw. Alle nutzen die gleiche E-Mail-Infrastruktur!

\section{Welche Aussage hat eine E-Mail- Adresse des Absenders?}

Genau genommen keine! Der Ferrero-Werbespruch von 1979 „Nur wo nutella draufsteht, ist auch nutella drin“ ist für E-MailAdressen leider nicht zu nutzen. Jeder ist im Prinzip in der Lage, für jede Domäne einen Namen eintragen zu lassen, wenn er noch nicht vergeben ist. Ob die E-Mail Adresse michael.schumacher@gmx.de, dem bekannten Rennfahrer Michael Schumacher gehört oder jemand anderem, z.B. Hape Kerkeling, können wir der E-Mail-Adresse nicht ansehen. Aber auch wenn wir wissen, wem eine E-Mail-Adresse wirklich gehört, hilft das nicht sehr viel, da jeder andere im Internet prinzipiell jede EMail-Adresse in seinen E-Mail-User-Agent als Absender-Adresse eintragen kann. Also eine Absender-E-Mail-Adresse hat keinen großen Wert, bezüglich der Verifikation der Echtheit des Senders. Für uns bedeutet das, dass wir nur zuverlässig den Absender einer E-Mail verifizieren können, wenn wir in der Lage sind, dies über den Inhalt der E-Mail zu tun oder wenn der Absender die E-Mail digital signiert hat und über ein gültiges Zertifikat verfügt.

\section{Welche Garantie gibt mir der E-Mail-Provider?}

Genau genommen keine! Der E-MailProvider sendet die E-Mail an einen anderen Provider, der die Domäne des Empfängers verantwortet. $\mathrm{Ob}$ die E-Mail zum richtigen Mail-Sever gesendet wird und dort tatsächlich in die Mail-Box des gewünschten Empfängers kommt, wird nicht und kann nicht garantiert werden. Die Zuverlässigkeit des Versandes liegt in der jeweiligen Verantwortung der TeilKomponente der E-Mail-Infrastruktur. Und hier hängt die Zuverlässigkeit von der richtigen Konfiguration und dem Betrieb des jeweiligen Mail-Servers und den DNS-Einträgen ab. Da es keine zentrale Instanz gibt, die eine „richtige“ Konfiguration konkret vorgibt und kontrolliert, hängt die Zuverlässigkeit vom Knowhow und der Sorgfalt der Betreiber ab. Da das notwenige Knowhow sehr groß und vielfältig ist, gibt es hier potentielle Risiken, die die Zuverlässigkeit schwächen.

\section{Wie kann ich die Echtheit des Inhaltes einer E-Mail überprüfen?}

Gar nicht! Es gibt sehr viele Stellen und Möglichkeiten, eine E-Mail während der Übertragung durch das Internet zu manipulieren. Wenn eine E-Mail nicht digital signiert wurde, kann der Inhalt beliebig während der Übertragung durch Angreifer verändert werden. Der Empfänger der E-Mail hat keine Möglichkeit festzustellen, ob der Inhalt während der Übertragung durch Dritte manipuliert worden ist. Daher ist die Überprüfung der Integrität nicht zuverlässig möglich.

\section{Wie zuverlässig ist die Vertraulichkeit des Inhaltes meiner E-Mail?}

Gar nicht! Wenn der Benutzer nicht explizit die Verschlüsselung der E-Mail vornimmt, wird zurzeit die E-Mail i.d.R. im Klartext zwischen den Mail-Servern durch das Internet gesendet. Das Internet besteht zurzeit aus mehr als 30.000 Autonomen Systemen (ASs), das sind unabhängige IPNetze, die zusammen das Internet bilden. Wie sich diese Autonomen System mitei- nander verbinden ist sehr unterschiedlich und hängt vom Charakter und der Strategie eines ASs ab. Formal gibt es die Möglichkeit, über einen Upstream Zugang zum gesamten Internet von den großen Autonomen Systemen zu kaufen oder mit Hilfe von Public und Private Peerings den $\mathrm{Zu}$ gang zu Teilen des Internets, für die Sender und Empfänger ASs kostenneutral, zu vereinbaren. Wie eine E-Mail von einem MailServer zum anderen Mail-Server gelangt, hängt von der Strategie der beteiligten AS ab. Hier wird bei akzeptabler Qualität i.d.R. der kostengünstigste Weg ausgesucht. $\mathrm{Ob}$ also eine E-Mail durch ein Land geleitet wird, in dem offiziell jede E-Mail analysiert und wirtschaftlich und politisch ausgewertet wird, können wir nicht direkt beeinflussen. Auch können Angreifer rechtswidrig die E-Mail in unserer Nähe (z.B. Hausanschlussraum im Bürogebäude) oder sonst wo im Internet mitlesen und zu ihrem Zwecke auswerten und nutzen.

\section{StatusQuo der E-Mail Zuverlässigkeit}

Wenn wir die Spam-Mails und Mails mit Malware sowie das Problem des potentiellen Mitlesens der E-Mails nicht betrachten, funktioniert der E-Mail-Dienst in den meisten Fällen „gefühlt“ zuverlässig, obwohl wir prinzipiell keine Garantie haben. Die Kommunikationsmechanismen sind in der Regel robust implementiert, d.h. Fehler werden durch die Fehlermechanismen in den beteiligten Kompetenten oder von den beteiligten Kommunikationspartnern entdeckt und durch den nochmaligen Versandt einer E-Mail kompensiert.

\section{Die Zukunft der E-Mail Zuverlässigkeit}

In unserer vernetzten Informations- und Wissensgesellschaft ist der E-Mail-Dienst so wichtig geworden, dass wir dafür sorgen müssen, dass die Zuverlässigkeit der E-Mail-Anwendung deutlich vertrauenswürdiger gestaltet werden kann. Vertraulichkeit, Integrität und Verbindlichkeit sind Eigenschaften, die zuverlässig gewährleistet werden müssen, damit die E-Mail-Anwendung weiterhin in unseren Geschäftsprozessen genutzt werden kann. 